\title{
The Effect of Self-Efficacy on Online Learning Efficiency in Cross-Cultural Perspective
}

\author{
Lei Ding ${ }^{1, a, *, \dagger}$, Ying $\mathrm{Zhu}^{2, \mathrm{~b},{ }^{*}, \dagger}$
}

\author{
${ }^{1}$ University of Glasgow, Glasgow, Scotland \\ ${ }^{2}$ University of Science and Technology Beijing, Beijing, China \\ *Corresponding author.Email:"a2629876D@student.gla.ac.uk, b41809097@xs.ustb.edu.cn
}

These authors contributed equally.

\begin{abstract}
Self-efficacy, a popular theme that is vital to influence learning efficiency. While online learning is another hot issue especially when the COVID-19 appeared. Therefore, understanding the distinction of self-efficacy on online education in different cultures and improving it has become important. In this review, self-efficacy's impacts on online learning efficiency in different cultures became the review's focus. There were different levels of autonomy related to making decisions in various cultures. And inner motivation seemed to be distinguished by cultural characteristics. Self-efficacy, possibly related to autonomy and motivation, seemed different in distinctive cultures during online courses as well. And self-regulation, a capability to achieve goals, was closely related to self-efficacy. So more research was referenced to show how to improve self-efficacy through intervening self-regulation. This review was mainly concentrated on applying scaffolding and training student's self-management, which probably influence the self-regulation from the western practices. In the current study, there were generally cross-sectional studies on self-efficacy, which can only focus on the fixed phase of participants' learning conditions. What's more, existing research was normally concentrated on the direct influence of self-efficacy that can only learn the difference of online learning outcomes on intrinsic factors. For further studies, they should do more intervention studies on self-efficacy in Eastern culture. Furthermore, the research on self-efficacy needs to conduct more longitudinal studies to observe changes of self-efficacy' impacts in the same subject. Moreover, adding more investigation on the outside environment's effects is needed.
\end{abstract}

Keywords: Self-efficacy, Online learning efficiency, Cross-cultural, Self-regulation.

\section{INTRODUCTION}

It is essential to understand self-efficacy to improve the quality of learning and working efficiency. Selfefficacy is, according to psychologist Albert Bandura, a person's confidence or belief in his or her skill to achieve a goal or result in a certain situation or a particular area [1]. Self-efficacy, a central content of the social-cognitive theory, has been studied in more than 10,000 investigations in the past 25 years. In industrialorganizational (I-O) psychology, self-efficacy has been remarked as a vital variable as well. In 1989, Landy named self-efficacy "the wave of the future" in work motivation research. Judging by the popularity in the field in the past years, Landy's prevision has been confirmed by the statistics [2]. What's more, self-efficacy was an important facility to cope with the increased stress of students when faced with a large number of academic requirements and to weaken the negative impacts of stressors. High levels of self-efficacy are associated with goal establishment, persistence, and a constructive approach to failures [3]. In addition, self-efficacy enables individuals to believe in their capabilities and to confront stressful requirements with confidence [4]. From the above, self-efficacy is very important to study, and cultures can affect the level of self-efficacy. Therefore, by understanding differences in self-efficacy in different cultures, more suitable interventions can be designed.

Nowadays, there are two learning theories, cognitive load theory (CLT) and constructivism, dealing with efficient instructional strategies in the recent literature. The definition of instructional efficiency is training with little cognitive load and brilliant performance results [5]. Cognitive load theory emphasizes that instruction guidance which facilitates learning efficiency is most 
beneficial [6]. Based on the assumption that information allowed by human cognitive architecture to be processed in working memory is limited, CLT demonstrates that information beyond the ability can enter working memory but cannot be encoded into long-term memory. CLT is composed of three types of cognitive load: intrinsic cognitive load, which is influenced by the content itself. The extraneous cognitive load, which is affected by irrelevant information and germane cognitive load, is dedicated to developing schemas (i.e., thought or behaviour modes that organize categories of information and their relationships). Constructivism emphasizes the significance of a conceptual understanding of the content. Constructivists support that novel information should be encoded actively and profoundly by learners, integrating it with their previous knowledge and experience and promoting deep learning. Several instructional approaches belong to constructivism, such as problembased learning (PBL), which effectively solves problems by integrating information. In PBL, learners solve problems through direct instruction and scaffolding [7]. Direct instruction involves that teacher provides materials within learners' capacity and demand. Scaffolding means providing assistance that is slightly beyond their ability to reach their proximal zone. According to the recent research, efficiency was improved by CLT rather than the constructivist approach, especially in the aspects of knowledge acquisition and retention. CLT and constructivist approaches were the same from the perspective of knowledge types. However, CLT approaches proved to be more efficient not only immediately but also one week later.

The previous study mainly focused on researching the impact of CLT (i.e., the most beneficial way is to motivate efficient learning and constructivist approaches) emphasizes the importance of obtaining effective learning methods on deep learning on acquiring knowledge effectively within computer-based learning progress based on a stimulation-based training (SBT) system. Self-efficacy is an important factor in learning efficiency. When it is put under the background of continuous globalization and the appearance of COVID19 , online learning becomes more and more popular and important to study. However, learning online and offline have various differences. For instance, face-to-face classes have peer support on learning objects, while online classes commonly need people to study independently. Hence, it is more vital to study the influence of self-efficacy on online learning efficiency. Yet, surprisingly limited researches have investigated the influence of self-efficacy on online learning efficiency, especially on cross-cultural perspectives, as previous studies mainly focused on one country or culture. Accordingly, this review aims to evaluate the related evidences or researches about the effect of self-efficacy on online learning efficiency in the context of eastern and western cultures. And in the current paper will mainly focus on previous studies which are investigating the self-efficacy of undergraduate students in different cultures who had online education, which may influence the learning efficiency, and the review will examine the previous studies to compare the differences of selfefficacy's effects between eastern and western cultures.

\section{CROSS-CULTURAL DIFFERENCES IN SELF-EFFICACY AND RELEVANT FACTORS}

Compared with individualism (i.e., the moral stance and ideology that support the value of the independence and self-reliance and advocate precedence of the interests of the individual) of western society, collectivistic values, which include Confucian teaching and authority of parents or senior people, were found to affect the learning and behavioural orientation of Asian students [8]. Considering the features of individualism and collectivism, western learners are more likely to have a high autonomy level (i.e., the ability to make an uncoerced, informed decision). At the same time, eastern students are likely to have a relatively low autonomy level and lack the chance to express their own opinions and questions. High autonomy demonstrates that learners can easily arrange their learning time and find proper approaches to learn new materials, playing a crucial role in online learning. However, Asian students, non-native learners with low autonomy levels and low capacity for critical thinking, are expected to encounter difficulties when taking online learning courses. This study conducted by Luyt demonstrated pedagogical structures dominated by Western educational practices and challenges that non-native students face [9]. In western education, online learning technologies facilitate selfdirection by giving students access to information and providing them with opportunities to decide their learning. Teachers hold the role of mentors and facilitators of knowledge instead of knowledge sources, which encourage collaboration and interaction to propel reflection and profound thinking. Under this background, non-native students encounter several difficulties. Nonnative students have to overcome challenges caused by constructive learning methods. It is an arduous task for non-native students to follow Western standards when thinking and writing and to adjust to settings in culture, linguistics, and institutions. Non-native learners relatively have poor performance when facing collaboration between students, the interaction between teacher and students, and critical thinking. In view of western students' high autonomy and eastern learner's low autonomy, respectively caused by individualism and collectivism, western students benefit more from online learning. In contrast, eastern students have to overcome several difficulties.

According to what was mentioned above, autonomy is expected to be different in various cultures and have 
relations with self-efficacy. What's more, inner motivation is related to self-efficacy as well. Likely, inner motivation (i.e., a driving force that comes from the intrinsic world, like curiosity) of online learners is distinctive in various cultures. The research demonstrated American online students' and Korean online students' different opinions about learning motivation [10]. Learning motivation involves six types, self-efficacy, affect, reinforcement, course relevance, learner control, and interest included. Online learners in America and Korea completed three self-report questionnaires. As a result, American online students showed a higher motivation mean score than those in Korea. There are significant mean differences between the two countries in five types of motivation. Learner control is the only one that Korean students scored higher while American students scored higher for the remaining, including course relevancy, course interest, reinforcement, self-efficacy. Cultural differences could be one of the reasons for the results. Western culture emphasizes thinking processes and learner's psychological characteristics, while Asian culture concentrates on collectivistic values. Asian students underline effort and performance goals while American learners stress the learning process itself and mastery of learning [11]. The reason for American students' high level of self-efficacy might also be their orientation for concentration on mastery and understanding materials and knowledge [12]. Based on cultural elements, western students are more likely to be stimulated by inner motivation. In contrast, eastern students might be motivated by the outside world, as a result of which western learners could perform well in online learning, and eastern learners might have poor performance in online education.

A larger number of differences exist in online learning in various cultures, such as inner motivation, difficulties in studying students are likely to be faced with, and learning approaches. Western learners are more likely to be driven by inner motivation and gain a strong sense of achievement. In contrast, eastern students are probably driven by motivations from the outside world, such as parents' rewards or social expectations. Motivation and autonomy are likely to exert distinctive influences on the self-efficacy of online learning in different cultures. The study identified the important factors influencing online learning based on learners' relationship with Hofstede's cultural model and their perceptions [13]. Researchers asked students from America, Spain, China, and Mexico who were categorized according to autonomy levels to finish two surveys. The first one measured self-efficacy and motivation, and the other measured learner support. Before the survey, Students from Spain and America were divided into the group of high autonomy, and learners from China and Mexico belonged to the low autonomous group. Students from Spain and America have the highest level of motivation and self-efficacy, followed by students in China and Mexico, considering that China and Mexico are countries with high power distance. Accordingly, students with low autonomy have less motivation and self-efficacy than those who feature high autonomy. Learners in Mexico scored higher in indulgence, as a result of which they have less motivation to begin an online course. In view of the positive correlation between motivation and self-efficacy, students in Mexico have a low level of self-efficacy. Cultural differences such as the power distance, individualism, pragmatic, and indulgence dimensions possibly contribute to the differences of learner factors. Western countries such as America and Spain are categorized in the group of high autonomy level while eastern countries such as China are divided into the low autonomy group. Considering that self-efficacy positively correlates with autonomy, western students with high self-efficacy can adapt to online education. Accordingly, eastern students with a low level of selfefficacy are likely to encounter adversities when taking online courses, such as lower efficiency and more distraction.

Because Korean students live in a more collectivistic society than American learners, they show more dependence on social groups. It is more likely that Korean students' level of self-efficacy and confidence is different from US learners. However, American students who have high self-efficacy are easy to acquire knowledge through online education. Based on American students' high level of self-efficacy, online education propels their positive behavioural intention toward online learning acceptance and satisfaction. US students attach great importance to self-efficacy and learning autonomy, while Asian learners probably seldom pay attention to them. Thus, people must understand whether it is more important in eastern culture to intervene self-efficacy when mentioning online learning. The research showed that the difference between American and Korean students influences perceived self-efficacy on ease of use and usefulness of online learning systems and its effects on student satisfaction [14]. Students in America and Korea completed a survey. From the results, in the perception of self-efficacy, US students scored higher than Korean students. Totally, the US students had higher scores than the Korean students in satisfaction. Korean students, compared to US students, support that selfefficacy plays a more crucial role in positive behavioural intention toward online learning acceptance and satisfaction. In contrast, online learning systems' ease of use insignificantly relates to behavioural intention toward online learning acceptance. The results indicated online learning self-efficacy positively influences online learning acceptance. Western students who grow up within individual values have a high level of self-efficacy. In contrast, eastern students depend more on social 
groups, lack enough self-efficacy, in which it is necessary to emphasize eastern learners' self-efficacy.

\section{APPROACHES TO PROMOTE ONLINE LEARNING EFFICIENCY THROUGH IMPROVING SELF-EFFICACY AND RELEVANT ABILITIES}

According to the above discussions, it has been shown that students living in western culture were more likely to have a higher self-efficacy than eastern students when they have online courses. To improve the selfefficacy of eastern students, understanding other factors influencing online learning efficiency and self-efficacy becomes particularly significant. Self-regulation (i.e., the people's ability to control the process of action and thought to achieve several goals) was an ability to achieve personal goals, maybe in connection with selfefficacy, which was a belief in achieving goals. Next, there is some literature to find whether there will be a stronger relationship between them. Pellas assessed the influence of metacognitive self-regulation, self-esteem (i.e., a special perspective that compromises each individual's value and postulates his/her self-evaluation and self-description in various aspects), and computer self-efficacy working on students' engagement factors (e.g., emotional-the students' interests and attitudes in the class; cognitive-how much did students put effort of intelligence into learning a project) in a large group of graduate and postgraduate students from the western countries (e.g., the United States, United Kingdom, Australia) with a set of questionnaires [15-17]. The results indicated that computer self-efficacy, self-esteem, and meta-cognitive self-regulation were positively associated with emotional and cognitive engagement factors. What's more, students who have higher selfefficacy, self-esteem, and self-regulation in an online program in Second Life were likely to be more engaged in online learning and have better outcomes cognitively and meta-cognitively. As self-regulation is closely linked to self-efficacy, they are both essential factors for online learning efficiency.

The above studies indicated that self-regulation could positively correlate with students' self-efficacy, and they both affect online learning efficiency among western countries. The above studies showed that students under the eastern culture generally demonstrated a lower level of self-efficacy, which is an important factor to learning online effectively. Therefore, it needs to further understand the association between the self-efficacy and self-regulation of online learners, especially in terms of eastern countries. Thus it will help us develop the training that improves self-efficacy from a cross-cultural perspective. In the study by $\mathrm{Su}$ et al., the relationship between online self-regulation and English language selfefficacy was assessed using questionnaires in a convenience sample of first-year undergraduate students (around 18-19 years old) in a Chinese university [18]. Understanding how self-regulation is correlated with self-efficacy is vital for English language education [19]. They found that the self-efficacy of EFL (learning English as a foreign language) learners is positively related to self-regulation in online learning. Through effective self-evaluation, which is a critical factor for improving students' English self-efficacy, EFL learners can become more clear about their learning goals and be more confident to speak and write English. In addition, EFL learners with more environment structuring (i.e., to find a proper environment that can help learners concentrate on studying and improve their online learning efficiency, also a significant predictor for English language self-efficacy) strategies during an internet-based learning process likely to be more selfefficacious in English speaking and writing. Researchers claim that self-efficacy and self-regulation are closely associated in that a high degree of self-regulation strengthens learners' beliefs in academic efficacy [20]. Thus, self-regulation and self-efficacy are both likely to have a positive relationship with online learning outcomes under the eastern culture.

Because there was limited literature about the eastern experiments on training self-efficacy, relevant western studies might be able to provide some guidance about how to effectively improve self-regulation. And from the previous study, it is known that increasing self-regulation is likely to enhance self-efficacy. The following research shows how to improve self-regulation by providing effective scaffolding (i.e., in an educational context, teaching tools or techniques to give a supporting foundation to the student on learning). Delen and colleagues conducted a study about the effects of interactive and instructional scaffolding on learning by comparing self-regulation and learning outcomes in traditional learning environments and the enhanced video environment in the sample of undergraduate and graduate students at a university located in southern Texas in the United States by putting forward an experimental design [21]. They found that the enhanced video learning environment was more functional and useful than the traditional learning environment in instruction in terms of students' learning performance. In addition, students were more engaged with online video-based instruction with purposeful interactive functions, therefore, remembered more information and have better learning performance. Meanwhile, graduate students had better self-regulatory behaviors in the enhanced video environment in their self-regulation strategies by taking more interactive notes. What's more, it seems that scaffolding could be a significant element that influences self-efficacy.

As scaffolding is an important factor, it is important to understand whether there is a cross-cultural difference. According to the findings from Yantraprakorn and his colleagues, who did the study on investigating learners' 
self-efficacy with their online learning skills and writing skills and how scaffolding improved both types of selfefficacy in the sample of an English tutorial school in Thailand, it shows that the students having distance language learning courses had low self-efficacy on writing before learning. Still, the supported scaffolding in the courses can improve the students' beliefs on that they could accomplish the tasks by themselves [22]. Rochanavibhata and Marian compared the scaffolding of teaching children between American and Thailand mothers, and the findings showed a cultural difference in using scaffolding strategies. It indicated that American mothers preferred to utilize various strategies, while Thailand mothers generally focused on a repetitive style which only using one scaffolding technique [23]. The results suggested that scaffolding the new learners in the area of self-efficacy on the distance writing education is valuable because it helped students enhance understanding in the learning process and deal with academic difficulties by helping to build students' experience and understanding of learning themes, the content, and offering successful experiences of mastering learning tasks. What's more, there were some differences in scaffolding between western and eastern cultures. As scaffolding is an essential factor in self-regulation on online education and has differences in different cultures, which is likely to be ignored in the eastern culture, enhancing the utilization of scaffolding (e.g., taking interactive notes, providing cohesive techniques for new learners) on online courses is likely to improve the selfregulation and self-efficacy of eastern students.

After referring to the Western research about training the students' self-efficacy by improving their selfregulation, another study in developing students' selfregulation and self-direction in online learning courses can be adopted to improve the eastern online learning efficiency. Stephen and Rockinson-Szapkiw put forward an experimental study to find the differences of selfdirected learning, online self-regulated learning, and online learning self-efficacy between a traditional FirstSemester Seminar (FSS) class and an experimental highimpact FSS class in the sample of undergraduate students from the United States [24]. FSS class in this paper was aimed to let undergraduate students adapt to college-level online learning, which was structured into four modules (i.e., module 1: introduction to research; module 2: choose a research topic and explore background research; module 3: doing research and finding sources; module 4: the value of information), and the student outcomes mainly focused on critical thinking, study skills, time management, and study habits. For example, students built a learning and classwork schedule, used and evaluated the impacts of reading, note-taking, time management, and writing techniques, sought counseling from support resources and systems, set targets, and assessed their commitment to their goals. This example includes the application of scaffolding, self-monitoring, and self-management, and to engage these factors during the learning process, self-regulation asks students to train a sense of self-efficacy. It showed that students in the high-impact FSS class significantly had better selfdirected learning and higher self-regulation than students in the traditional FSS class. While the online learning self-efficacy was higher in the high-impact FSS class than the traditional class, there was no statistically significant difference between the two types of class on the online learning self-efficacy. Moreover, online learning self-efficacy supported students' development of self-regulation and self-direction. Therefore, for the eastern students, the application of scaffolding, selfmanagement, and self-monitoring (e.g., building a learning schedule, managing their time, self-review and feedback) is essential to improve their self-regulation, accordingly to enhance their self-efficacy and online learning outcomes.

Overall, self-regulation is an essential factor for enhancing online learning efficiency, just like selfefficacy in Western and Eastern cultures. By drawing on western studies about improving self-regulation, there were some effective methods of strengthening eastern students' self-regulation and self-efficacy. One is to utilize scaffolding like taking notes, and the other one is to improve their self-management and self-monitoring. Accordingly, the online learning outcomes and selfefficacy will be enhanced.

\section{LIMITATION AND FUTURE DIRECTION}

The current studies mainly focused on correlational studies. For example, they researched the relation between self-efficacy and online learning or other relevant elements. Recent studies lack enough eastern intervention studies, in which eastern students usually learn from the western countries. Future studies should attach more importance to intervention studies in eastern cultures. In addition, most of the recent researches is cross-sectional researches between online learning and self-efficacy by controlling some variables like their ages or genders. Future studies must conduct longitudinal studies to understand how self-efficacy dynamically influences learning outcomes. It allows people to learn the relations between self-efficacy and online education in childhood and the dynamic changes. Ultimately, researches in recent years only concentrate on the direct impacts of self-efficacy and individual differences like self-regulation. Future studies should examine the influences of environmental factors such as parenting styles, resulting in a more comprehensive understanding of online education. It should stress the interactions between several factors as well. 


\section{CONCLUSION}

To conclude, students from western and eastern cultures generally have different performances during online courses where western students prefer to have more interaction, cooperation, and critical thinking than Asian students. It suggests that western students are easier to be encouraged by the inner motivation while eastern students' learning outcomes are more dependent on outside factors. Moreover, western students are likely to have higher autonomy than eastern sides, as eastern students generally have a lower motivation to have online courses. Accordingly, their self-efficacy comes to be lower as well. Then, it indicates that eastern students are probably to have a lower acceptance of online education. They are also more likely to be dissatisfied with online courses, which leads to their lower self-efficacy and worse online learning efficiency than western students.

To handle this gap between western and eastern students' self-efficacy in online learning, it is important to enhance self-regulation, which takes a similarly vital place and self-efficacy. From previous studies, it was found that self-regulation has a positive correlation with online learning outcomes. So improving self-regulation through effective methods to increase eastern students' self-efficacy on online learning is very important. According to the existing Western research, one useful tool is to use scaffolding, which can improve students' engagement during online classes. And another one is practicing students' self-management and selfmonitoring, which can increase their absorption and understanding of knowledge effectively on online learning. However, the current studies consist of correlational research, which lacks eastern intervention experiments, and compared with cross-sectional research in terms of online learning and self-efficacy, fewer longitudinal studies were conducted. Recent research only concentrates on individual differences in students' self-efficacy, which directly affects online learning efficiency. Therefore, it suggests that more intervention researches and tracing experiments on eastern sides need to be carried out, and the impacts of environmental elements on self-efficacy like parenting styles have to be included in future studies to have a more comprehensive perception in improving self-efficacy and online learning efficiency.

\section{REFERENCES}

[1]Bandura, A. (2010). Self-efficacy the corsini encyclopedia of psychology. John Wiley \& Sons, Inc., 1-3.

[2]Landy, F. J. (1989). Psychology of Work Behavior. Pacific Grove.
[3]Jerusalem, M., \& Mittag, W. (1995). Self-efficacy in stressful life transitions. Self-efficacy in Changing Societies, 177-201.

[4]Schwarzer, R. (2000). General Perceived Self-efficacy in 14 Cultures. Freie Universität Berlin.

[5]Sweller, J., Van Merrienboer, J. J., \& Paas, F. G. (1998). Cognitive architecture and instructional design. Educational psychology review, 10(3), 251296.

[6]Vogel-Walcutt, J. J., Gebrim, J. B., Bowers, C., Carper, T. M., \& Nicholson, D. (2011). Cognitive load theory vs. constructivist approaches: which best leads to efficient, deep learning?. Journal of Computer Assisted Learning, 27(2), 133-145.

[7]Loyens, S. M., \& Gijbels, D. (2008). Understanding the effects of constructivist learning environments: Introducing a multi-directional approach. Instructional science, 36(5), 351-357.

[8]Sue, S., \& Okazaki, S. (1990). Asian-American educational achievements: A phenomenon in search of an explanation. American psychologist, 45(8), 913.

[9]Luyt, I. (2013). Bridging spaces: Cross-cultural perspectives on promoting positive online learning experiences. Journal of Educational Technology Systems, 42(1), 3-20.

[10]Lim, D. H. (2004). Cross cultural differences in online learning motivation. Educational Media International, 41(2), 163-175.

[11]Grant, H., \& Dweck, C. S. (2001). Cross-cultural response to failure: Considering outcome attributions with different goals. In Student Motivation (pp. 203-219). Springer, Boston, MA.

[12]Salili, F., Chiu, C. Y., \& Lai, S. (2001). The influence of culture and context on students' motivational orientation and performance. In Student motivation (pp. 221-247). Springer, Boston, MA.

[13]Gómez-Rey, P., Barbera, E., \& Fernández-Navarro, F. (2016). The impact of cultural dimensions on online learning. Journal of Educational Technology \& Society, 19(4), 225-238.

[14]Lee, J. W., \& Mendlinger, S. (2011). Perceived selfefficacy and its effect on online learning acceptance and student satisfaction. Journal of Service Science and Management, 4(03), 243.

[15]Vrugt, A., \& Oort, F. J. (2008). Metacognition, achievement goals, study strategies and academic achievement. Metacognition and Learning, 3(2), 123-146. 
[16]Branden, N. (2001). The psychology of self-esteem: A revolutionary approach to self-understanding that launched a new era in modern psychology. JosseyBass.

[17]Pellas, N. (2014). The influence of computer selfefficacy, meta-cognitive self-regulation and selfesteem on student engagement in online learning programs: Evidence from the virtual world of Second Life. Computers in Human Behavior, 35, 157-170.

[18]Su, Y., Zheng, C., Liang, J. C., \& Tsai, C. C. (2018). Examining the relationship between English language learners' online self-regulation and their self-efficacy. Australasian Journal of Educational Technology, 34(3), 105-121.

[19]Kim, D. H., Wang, C., Ahn, H. S., \& Bong, M. (2015). English language learners' self-efficacy profiles and relationship with self-regulated learning strategies. Learning \& Individual Differences, 38, 136-142.

[20]Zimmerman, B. J., \& Martinez-Pons, M. (1992). Perceptions of efficacy and strategy use in the selfregulation of learning. Student perceptions in the classroom: Causes and consequences, 185-207.

[21]Delen, E., Liew, J., \& Willson, V. (2014). Effects of interactivity and instructional scaffolding on learning: Self-regulation in online video-based environments. Computers \& Education, 78, 312-320.

[22]Yantraprakorn, P., Darasawang, P., \& Wiriyakarun, P. (2013). Enhancing self-efficacy through scaffolding. In The Proceedings of the Third International Conference on Foreign Language Learning and Teaching, The Ambassador Hotel, Bangkok, Thailand.

[23]Rochanavibhata, S., \& Marian, V. (2020). Maternal scaffolding styles and children's developing narrative skills: A cross-cultural comparison of autobiographical conversations in the US and Thailand. Learning, Culture and Social Interaction, 26, 1-15.

[24]Stephen, J. S., \& Rockinson-Szapkiw, A. J. (2021). A high-impact practice for online students: the use of a first-semester seminar course to promote selfregulation, self-direction, online learning selfefficacy. Smart Learning Environments, 8(1), 1-18. 